\title{
Interview
}

\section{Julie Williams speaks to Personalized Medicine}

Julie Williams is a Professor at the Department of Psychological Medicine and Neurology at Cardiff University School of Medicine, Wales, UK. She is currently part of the Medical Research Council Center on Neuropsychiatric Genetics and Genomics where she heads the Neurodegeneration Section. Her research focuses on identifying and understanding genes that increase the risk of developing complex psychological and neurodegenerative disorders including Alzheimer's disease, developmental dyslexia and schizophrenia. In 2009, Professor Williams published evidence from a powerful genome-wide study for the first new susceptibility genes for Alzheimer's disease in 17 years. Along with results from a second genome-wide association study, these findings were highlighted by Time Magazine as one of the ten most important medical breakthroughs of 2009. Recently, Professor Williams led a group that discovered five gene variants that raise the risk of Alzheimer's disease, bringing the known number of genes associated with the disease to ten. With genes known to account for $20 \%$ of a person's chance of developing late-onset Alzheimer's disease, the cost in long-term care and lost productivity reaching $\mathrm{f} 23$ billion (according to a dementia report commissioned last year by the Alzheimer's Research Trust), understanding the genes responsible for the steady and irreversible destruction of brain cells leading to the disease is crucial.

- As an undergraduate student, what were you interested in?

My first degree was in Psychology, and then I went on to do my PhD in Psychology at Cardiff University, Wales, UK. What motivated me then was learning how the brain worked and I joined what is now the Medical Research Council center for neuropsychiatric genetics and genomics when it was first started approximately 20 years ago - my focus then was on schizophrenia.

While having initially worked in the field of schizophrenia, how did your research come to focus on Alzheimer's disease?

After a few years working on schizophrenia I became interested in Alzheimer's disease because I thought that is was something, that from a genetic point of view, was more advanced and a disease that we could learn from and use to identify genes for other disorders - thinking at the time that it would all be done and dusted very quickly! But for the last 15 years, I have concentrated on Alzheimer's disease and to a lesser extent on developmental dyslexia. What motivates me is understanding how this neurodegenerative process happens in the brain - it is obvious that this is a serious illness and the number of people with it is going to increase. It was something that had to be prioritized and thus an obvious thing to spend my time working on.

- What field of Alzheimer's disease research were you initially focused on and what was the field like back then?

My initial focus was mainly on common Alzheimer's disease, the form which occurs after the age of 65 years. We knew from 25 years ago that there were three genes that caused rare forms of earlyonset Alzheimer's disease and that has been a major focus of research ever since.

Approximately 19 years ago, the first gene that increased an individual's risk of developing the disease, $A P O E$, was discovered. This had quite a significant effect size on risk but it took us 17 years to actually identify the next susceptibility genes which we published in 2009 from a genome-wide association study (GWAS). This involved the pulling together of a large consortium of research groups from Europe, the UK and the USA. Together we published two new genes - CLU and PICALM - in the same journal as the results of a GWAS by a French group (headed by Philippe Amouyel) were published. Their top hit in clusterin was our top hit - that fitted

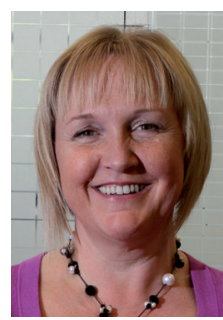

\section{Julie Williams}

Lower Ground Floor, Henry Wellcome Building, Heath Park, Cardiff University, School of Medicine, CF14 4XN, Cardiff, UK

Williams」@cf.ac.uk 
very nicely and, in addition, they got genome-wide significance for a third gene, compliment receptor 1 . When we put our datasets together after the papers had been published and we also included collaboration with a third consortium, the Cohorts for Heart and Aging Research in Genomic Epidemiology (CHARGE) Consortium, we identified a fourth gene BIN1, which was published in 2010 .

\section{How did you expand upon} these findings?

Moving on from there, we continued this collaboration and followed up some of the findings and genes that demonstrated very strong evidence but were not genome-wide significant. We tested them, knowing that there were more of these hits than would be expected by chance - it was a case of identifying which ones were genuine and which ones were not. We then took those genuine SNPs forward and tested them in thousands of other samples, from that we identified $A B C A 7$ and the MS4 cluster.

In the summer of 2010, we were approached by the Alzheimer's Disease Genetics Consortium (ADGC) as they had similarly just finished their recent GWAS with their top genome-wide significant hit coinciding with our MS4 finding. In a similar way they had identified four genes in their sample that demonstrated strong evidence but weren't genome-wide significant. Subsequently, they asked us to investigate those four genes in our dataset from which we were able to add evidence for three which took them over genomewide significant level. One of the genes fell away as we had no evidence in our samples of an effect. So with those combined efforts we were able to identify five new susceptibility genes.

\section{- Several Alzheimer's disease risk genes have previously been} implicated in the immune response, how do the five new genes you identified affect disease progression?

What is exciting is that the genes we found appear to cluster in potential pathways of which three can be pulled out. First, the immune response appears to be important - several of the genes implicate some inflammatory or immune response indicating that individuals who develop Alzheimer's disease have a slightly different immune response and that feeds directly into the disease process and affects risk. Immunity has been a focus of study ever since evidence of an immune response in the brains of original cases suffering from Alzheimer's disease were observed. We had always thought that this was just a passive response, but what these genes are telling us is that there is something different about this response that increases an individual's risk of developing the disease.

The second area that was implicated was cholesterol processing: $A P O E, A B C A 7$ and $C L U$ all have roles in the processing of lipids, including cholesterol in the brain. So these genes indicate that there may be something different about the processing of cholesterol that again feeds into disease risk.

\section{Were there any unexpected} findings from the study?

The most interesting and novel observation, I think, is that we now have four genes that implicate a very specific cellular process - endocytosis. This is the taking in of big molecules from outside the cell inside the cell and the subsequent processing and expulsion of them as by-products out of the cell. Our results suggest there is something different about this endocytosis process in those that are developing Alzheimer's disease. This is a very novel observation and should be a strong focus for future research to understand what exactly is different and how that is contributing to disease.

- By using next-generation genome sequencing in your next research steps, what are you hoping to achieve?

Some of the first things from a genetic point of view that you want to look at is have you got the correct signal? You may have a very strong signal in particular gene regions, but you need to ask is this the variant that is having the functional affect? Although most of these findings implicate gene regions, in very few cases is it obvious what the variant is likely to be doing. In addition, it is very likely that these effects may be subtle, such as differences in gene expression or different forms of the gene 
being expressed - it's not big mistakes in the coding regions that have very large effects on the individual. As a result, it is going to be a more complicated process to nail down exactly what the variant(s) within the gene regions are actually having a functional effect on and sequencing will allow us to get a better view on that.

If you sequence the regions that are showing correlation or linkage disequilibrium with the major SNP that is associated with the disease then you can not only get a better idea of the common variants that may be contributing to the disease, but also of possibly identifying rarer variants within the coding of genes that are having an effect on a smaller number of individuals, but are still contributing to disease. Sequencing will allow us to identify, although we will still need a large sample size - several hundred people - to nail down those effects.

What is currently known about the pharmacogenomics of Alzheimer's disease?

With regards to pharmacogenomics, the focus is still on manipulating amyloid $\alpha$ Tau. But with these new variants that are now focusing on specific functional pathways, there still remains a lot of work to be done in order to actually identify and understand how these gene variants actually contribute to the biology of the disease. This is not a tremendously difficult science, but it is something that needs to be done. Hopefully, from that we will be able to get drug targets with which we can manipulate and reduce risk.

\section{- Do you think that it is likely that} doctors may start prescribing preventative drugs for Alzheimer's disease?

Absolutely, I think it is highly likely that for the next generation, people will be taking a number of drugs to prevent or delay the onset of dementia much like we are currently taking statins and controlling our blood pressure to reduce the risk of heart disease.
What other research projects are you currently involved in?

I have developed a research group on developmental dyslexia. The next step in studying this disorder is to do a genome-wide association study - I just need funding for it! The beauty about this is that we can learn a lot more about the disease by simply identifying the genes involved. Like with all of these complex diseases, we don't need to know $100 \%$ of all the causes and risk factors to be able to have quite a significant effect on the disease if we can produce the appropriate therapy. This is a very exciting time; we are learning a lot, and what the genetics is telling us is actually quite a different story than many had thought up until now.

Where do you see your research being focused in the next 10 years? Ideally, we should now be increasing our funding of this sort of research because it takes at least 10 years to get drugs through clinical trials - we need to be working hard and efficiently and with adequate funding to try and understand what these new discoveries mean, because we know how long it takes to get a new drug into the clinic. We do need to expand our research base in dementia and attract basic researchers from different fields to work with us.

\section{Disclaimer}

This work is the opinion of the author and does not necessarily represent the views of Future Medicine or its employees.

\section{Financial \& competing interests disclosure}

The author is involved in patents relating to some of the susceptibility genes discovered. The author has no other relevant affiliations or financial involvement with any organization or entity with a financial interest in or financial conflict with the subject matter or materials discussed in the manuscript. This includes employment, consultancies, honoraria, stock ownership or options, expert testimony, grants or patents received or pending, or royalties.

No writing assistance was utilized in the production of this manuscript. 